\title{
Permeability and Clearance Views of Drug Absorption: A Commentary
}

\author{
Hans Lennernäs, ${ }^{1}$ John R. Crison, ${ }^{2}$ and Gordon L. Amidon ${ }^{2}$
}

Received December 14, 1994 -Final May 17, 1995

The commentary by Chiou (this issue) raised several questions and offered alternative points of view on the interpretation and limitations of the permeability concept in the biopharmaceutical discipline. We briefly review the approaches below and show that the calculated clearance per unit area and permeability are identical variables and the central point in such analysis is the choice of luminal reference drug concentrations. We further consider the effective permeability view as most appropriate because it provides a sound intuitive understanding of the physical and mechanistic principles underlying the transport process.

The starting point for analyzing drug transport across the membrane wall of a tube is the relationship between the mass entering and leaving the tube

$$
d M / d t=Q_{\text {in }} C_{\text {in }}-Q_{\text {out }} C_{\text {out }}=Q\left(C_{\text {in }}-C_{\text {out }}\right)
$$

where $C_{\text {in }}$ and $C_{\text {out }}$ are the inlet and outlet drug concentrations, respectively, and $Q$ is the flow through the tube, usually assumed to be constant. The mass balance relationship can then be set to describe the transport rate of the drug across the tube membrane (absorbed mass) according to Fick's first law

$$
d M / d t=A \cdot P_{\text {eff }}\left(C_{\text {ref }}^{\text {lumen }}-C_{\text {ref }}^{\text {blood }}\right)
$$

where $A$ is the surface area of the membrane, $P_{\text {eff }}$ is an effective permeability

\footnotetext{
'Department of Pharmacy, Division of Biopharmaceutics and Pharmacokinetics, Uppsala University, Uppsala, Sweden.

${ }^{2}$ College of Pharmacy, Department of Pharmacy, University of Michigan, Ann Arbor, Michigan.
} 


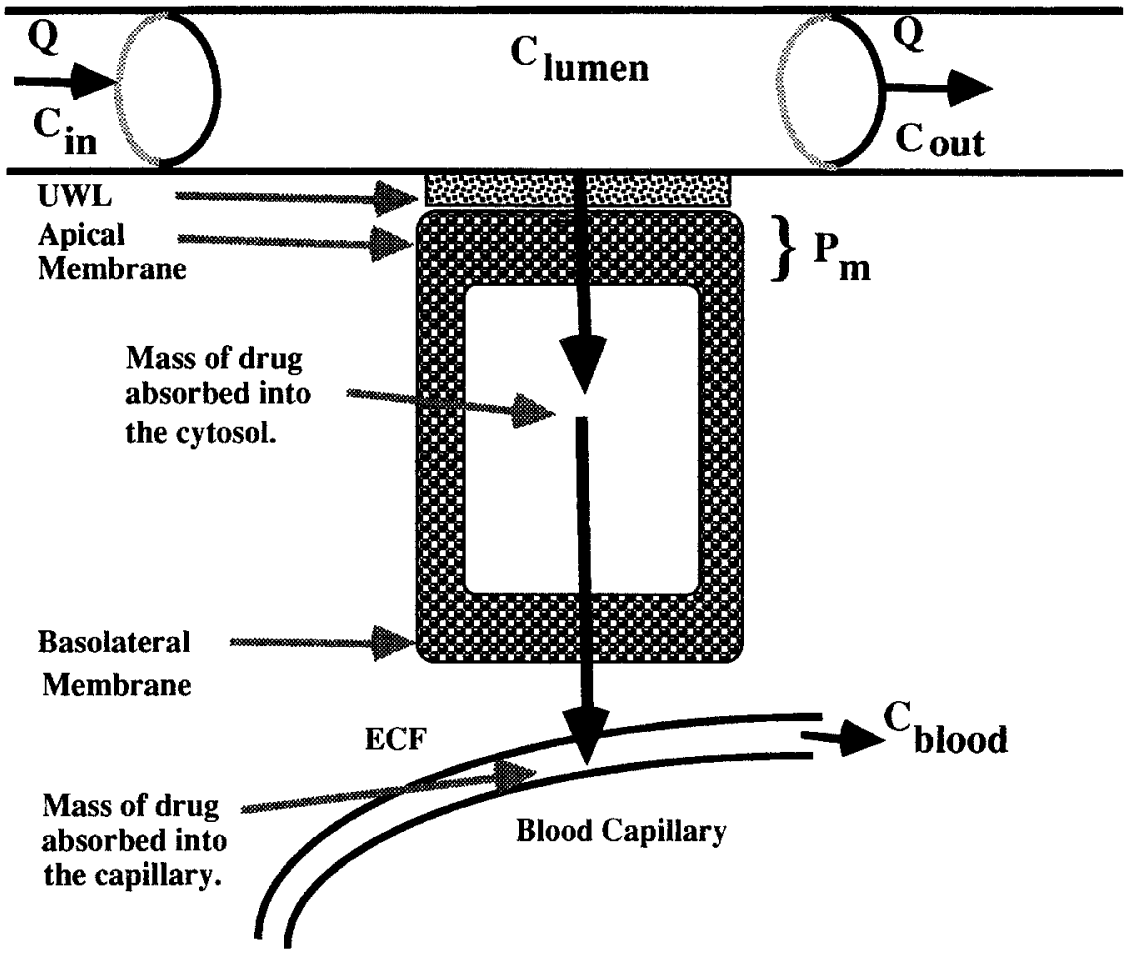

Fig. 1. Illustration of the different transport barriers a drug molecule has to pass during the intestinal absorption process (UWL: unstirred water layer, ECF : extracellular fluid, Pm: membrane permeability).

coefficient and the reference concentrations are on the two opposite sides of the intestinal mucosa; $C_{\text {ref }}^{\text {lumen }}, C_{\text {ref }}^{\text {blod }}$ (Fig. 1). It is usually assumed that the reference blood concentration $\left(C_{\text {ref }}^{\text {blood }}\right)$ is negligible in comparison with the lumen concentration. This has been directly shown to be valid for antipyrine in humans (1).

Alternatively, the approach of using a clearance concept is described by

$$
d M / d t=C l_{\mathrm{abs}} C_{\text {ref }}^{\text {lumen }}
$$

where $C_{\text {abs }}$ is the absorptive clearance $(\mathrm{ml} / \mathrm{min})$ into the body and $C_{\text {ref }}$ is the same reference concentration as used above. Using the same assumption for Eqs. (2) and (3), it is clear that $P_{\text {eff }}$ is equal to $C l_{\text {abs }} / A$, if the same reference concentration is used. Thus, the use of the term "effective permeability" or "absorptive clearance per unit area" is perhaps a matter of preference. However, we prefer the permeability view since it is based on 
fundamental mass transport principles, e.g., Fick's first law applied to a membrane and models for drug transport based on permeabilities have been extensively developed (2). An additional difficulty in using absorptive clearance itself is that it depends on surface area and as a result the clearance would depend on the length of the segment studied and would make comparisons between experiments and laboratories with slightly different techniques much more uncertain. Moreover, while clearance has had a wide application in general, it is most often used to describe drug elimination in pharmacokinetics. It has the unit of $\mathrm{ml} / \mathrm{min}$ and is often expressed as the virtual volume of fluid from which the substance is completely removed per unit time (3). The physical understanding of $C l_{\text {abs }}$ is therefore not as direct as for $P_{\text {eff }}$ in the more defined intestinal perfusion system.

The calculation of permeability or clearance per unit area each requires an estimation of membrane surface area [A, Eqs. (1) and (2)]. This has conventionally been taken to be the geometrical surface area based on the length and radius of the perfused segment. The normal intestine is, of course, not straight or rigid, this implies that the actual absorbing surface area effect is incorporated into the measured effective permeability. The "true" cellular membrane permeability $\left(P_{\mathrm{m}}\right)$ of the intestinal mucosa undoubtedly varies from villus crypt to tip and axially from stomach to colon. The effective permeability $\left(P_{\text {eff }}\right)$ will be an average over the segment perfused.

The need for a reference concentration is evident with either definition, and the available choices are the inlet, outlet, or some calculated average concentration. The choice is usually made based on which concentration more closely approximates the concentration adjacent to the intestinal wall $\left(C_{\mathrm{w}}\right)$. Ideally, one would have the concentration at each point along the absorbing surface, since Fick's law is a local law, which would provide us with "local" permeabilities. For some experimental systems where the hydrodynamics are well defined, the transport equations for the convective process and diffusion can be solved analytically $(2,4)$. However, the regional perfusion approach in the human intestine only offers entering and leaving drug concentrations. For this system we have determined, based on nonsteady-state analysis of tracer curves, that the hydrodynamics is described best by a mixing-tank model, for convection within a perfused $10-\mathrm{cm}$ long jejunal segment $(4,5)$.

The effective permeability includes the process of transport to the membrane (aqueous permeation, e.g., diffusion and convection to the membrane), cell mucosa permeation including mucin and membrane translocation processes (passive and/or active transcellular transport or passive paracellular diffusion/convection), and perhaps transport through the cytosol, basolateral membrane, interstitial fluid, and capillary wall to the blood (see Fig. 1) (6-8). However, it is assumed that the permeability is dominated by the 
largest resistance which is usually considered to be the apical brush border membrane. Drug metabolism in the cell cytosol may influence the measured permeability through further maintaining sink conditions intracellularly, and carrier-mediated exsorption of a drug will give a "lower" effective permeability. These processes certainly need to be accounted for in any detailed model of the drug transport mechanism across the mucosal membrane. Furthermore, the permeability is measured at steady state in the perfusion system so that the binding process that may influence (non-steady-state) drug permeation are quasi-steady state and do not contribute to the measured permeability (6). The human effective permeability has also been shown to correlate very well with the fraction absorbed in vivo for a broad range of structurally different compounds, which also supports the mass balance between disappearance and appearance rate $(9,10$, Lennernäs et al., to be published). In addition, we have compared human $P_{\text {eff }}$ values between the Caco-2 model and the rat perfusion model considering $P_{\text {eff }}$, and the usefulness of the permeability concept was evident $(11,12)$. Finally, drug instability in the intestinal lumen and/or brush border metabolism also influences the observed permeability but can be approximated by analyses of the metabolites in the perfusate and as well in vitro studies. However, these considerations apply to both permeability and clearance views.

In summary, the permeability view of the absorption process is the most fundamentally sound view upon which to base analysis of this complex process. The commentary by Chiou advancing the absorption clearance concept, while correctly noting many of these complexities, does not offer any real advance in analysis or understanding and may well add to the confusion in this field.

\section{REFERENCES}

1. H. Lennernäs, Ö. Ahrenstedt, R. Hällgren, L. Knutsson, M. Ryde, and L. K. Paalzow. Regional jejunal perfusion, a new in vivo approach to study oral drug absorption in man. Pharm. Res. 9:1243-1251 (1992).

2. R. L. Elliott, G. L. Amidon, and E. N. Lightfoot. A convective mass transfer model for determining intestinal wall permeabilities: Laminar flow in a circular tube. $J$. Theoret. Biol. 87:757-771 (1980).

3. G. R. Wilkinson. Clearance concepts in Pharmacology. Pharmacol. Rev. 39:1-47 (1987).

4. G. L. Amidon, J. Kou, R. L. Elliott, and E. N. Lightfoot. Analysis of models for determining intestinal wall permeabilities. J. Pharm. Sci. 69:1369-1373 (1980).

5. I-D. Lee, U. Fagerholm, H. Lennernäs, and G. L. Amidon. A study of hydrodynamic characteristics in human intestine applying residence time distribution analysis. In $U$. Arto (ed.), Symposium on Methods to Overcome Biological Barriers in Drug Delivery, August 26-28, Kuopio University Publications A (Pharm. Sci.), Vol. 10, 1993, p. 73.

6. E. L. Cussler. Diffusion, Mass Transfer in Fluid Systems, Cambridge University Press, Cambridge, England, 1984.

7. W. D. Stein. Channels, Carriers, and Pumps: An Introduction to Membrane Transport, Academic Press, New York, 1990. 
8. W. D. Stein. Transport and Diffusion Across Cell Membranes, Academic Press, New York, 1986.

9. H. Lennernäs. Gastrointestinal absorption mechanisms: A comparison between animal and human models. Eur. J. Pharm. Sci. 2:39-43 (1994).

10. G. L. Amidon, H. Lennernäs, V. P. Shah, and J. Crison. Theoretical considerations in the correlation of in vitro drug product dissolution and in vivo bioavailability: A basis for a biopharmaceutics drug classification. Pharm. Res. 12:413-420 (1995).

11. H. Lennernäs, K. Palm, U. Fagerholm, and P. Artursson. Comparison between active and passive drug transport in human intestinal epithelial (Caco-2) cells in vitro and human jejunum in vivo. Int. J. Pharm. in press, (1995).

12. U. Fagerholm and $H$. Lennernäs. The correlation between rat and human small intestinal permeability to drugs with different physico-chemical properties. Pharm. Res. 11:S263 (1994). 